\title{
Radiation Safety Certification: A Review
}

\author{
Cybil J. Nielsen \\ Nuclear Medicine Technology Program, Department of Radiologic and Imaging Sciences, Indiana University School of Medicine, \\ Indianapolis, Indiana
}

CE credit: For CE credit, you can access the test for this article, as well as additional JNMT CE tests, online at https://www.snmmilearningcenter.org. Complete the test online no later than December 2021. Your online test will be scored immediately. You may make 3 attempts to pass the test and must answer $80 \%$ of the questions correctly to receive $1.0 \mathrm{CEH}$ (Continuing Education Hour) credit. SNMMI members will have their CEH credit added to their VOICE transcript automatically; nonmembers will be able to print out a CE certificate upon successfully completing the test. The online test is free to SNMMI members; nonmembers must pay $\$ 15.00$ by credit card when logging onto the website to take the test.

It has become common for nuclear medicine technologists to assume the responsibilities, or even the role, of the radiation safety officer or associate radiation safety officer. Their responsibilities are primarily related to the radioactive materials license but increasingly can include additional safety responsibilities within the hospital. These include CT, MRI, and fluoroscopy safety. Many technologists reading this article may be interested in sitting for the radiation safety advanced certification examination by the Nuclear Medicine Technology Certification Board. A consultation of the content outline for that examination (found on the Nuclear Medicine Technology Certification Board website) is a good place to start. The content outline is quite extensive and cannot be covered within a single article.

Key Words: radiation safety officer; nuclear medicine technologist; Nuclear Regulatory Commission

J Nucl Med Technol 2018; 46:321-325

DOI: $10.2967 /$ jnmt.118.209528

$\mathbf{T}$ he purpose of this article is to provide a brief summary of some of the knowledge required for technologists to assume the role of a radiation safety officer (RSO) or associate RSO. A good place to start is to review the following Nuclear Regulatory Commission (NRC) regulations: NRC Title 10CFR19: Notices, Instructions, and Reports to Workers; NRC Title 10CFR20: Standards for Protection Against Radiation; NRC Title 10CFR35: Medical Use of Byproduct Material; and NRC Title 10CFR71: Transportation of Radioactive Material.

Facilities are bound by what is in their application for the radioactive materials license. When the license application is initially submitted either to the NRC or to the state (if in an agreement state), it may contain items that are stricter

\footnotetext{
Received Aug. 29, 2018; revision accepted Sep. 25, 2018.

For correspondence or reprints contact: Cybil Nielsen, Indiana University School of Medicine, 1120 W. Michigan St. CL-120, Indianapolis, IN 462025111.

E-mail: cybniels@iupui.edu

Published online Nov. 9, 2018

COPYRIGHT (c) 2018 by the Society of Nuclear Medicine and Molecular Imaging.
}

than the regulations require. A technologist should always consult the facilities' radioactive materials license application before beginning work at a new facility. Annual refresher training for occupationally exposed workers should include these license-specific items in radiation safety $(I)$.

Within the NRC regulations, licensing information can be found, as well as training and experience requirements for RSOs. The pathway for technologists to become RSOs includes $200 \mathrm{~h}$ of didactic training (usually obtained in nuclear medicine school) and $1 \mathrm{y}$ of full-time radiation safety experience under the supervision of an existing RSO. This training and experience must have been obtained within the $7 \mathrm{y}$ preceding the date of application, or the individual must have had related continuing education (1).

The transportation of radioactive material is regulated by the Department of Transportation; however, the NRC can (and will) enforce those regulations. This includes hazardousmaterials training for anyone involved in transporting radioactive materials. Hazardous-materials training needs to be done every 3 y (2). Radioactive packages need to be labeled as white I, yellow II, or yellow III (Table 1) (3). Incoming radioactive packages must be checked in within $3 \mathrm{~h}$ of receipt (4). Incoming and outgoing radioactive packages require dose rate and removable contamination surveys to be performed (1). When a package is returned to a radiopharmacy, it is labeled as an "excepted" package and only limited quantities of radioactive material can be included (5). If the limited quantity is exceeded, the department should allow for decay before returning the package. One common confusion among RSOs is the difference between exempt quantities and limited quantities. An exempt quantity is the amount of each radionuclide that you can obtain without a radioactive materials license. A limited quantity is the quantity of material that can be shipped in an excepted package (5).

\section{PHYSICS REVIEW}

The atom is made of a nucleus (protons and neutrons) and an electron cloud. An element, such as technetium or iodine, is the identity of the atom. The number of protons in the nucleus determines this identity (or elemental name). 
TABLE 1

Package Labels for Radioactive Materials Packages

\begin{tabular}{lcc}
\hline Label & Surface limit & Transport index $(1 \mathrm{~m})$ \\
\hline White I & $0.5 \mathrm{mR} / \mathrm{h}$ & Not applicable \\
Yellow II & $50 \mathrm{mR} / \mathrm{h}$ & $1 \mathrm{mR} / \mathrm{h}$ \\
Yellow III & $200 \mathrm{mR} / \mathrm{h}$ & $10 \mathrm{mR} / \mathrm{h}$ \\
\hline
\end{tabular}

This number of protons in the nucleus is the atomic number, denoted by the letter $\mathrm{Z}(6)$.

If an atom is unstable, it is said to be radioactive. It will emit radiation (energy) until it becomes stable again. The amount of time it is unstable can be measured in half-lives (the time it takes for half the energy to decay). There are 3 modes of radioactive decay originating from an unstable nucleus: $\alpha$, isobaric ( $\beta$ or electron capture), and isomeric ( $\gamma$-emission or internal conversion). $\alpha$ - and $\beta$-decay are particulate decay. An $\alpha$-particle is simply a helium nucleus ( 2 protons and 2 neutrons). A $\beta$-particle is an electron and can be either positive or negative. Isobaric decay can either be $\beta$-decay or electron capture and occurs when the nucleus is either proton-rich or neutron-rich. $\beta$-positive decay occurs when the nucleus is proton-rich, and $\beta$-negative decay occurs when the nucleus is neutron-rich. Electron capture is an alternate decay pathway to $\beta$-positive decay. If a nucleus is proton-rich but does not have enough energy for $\beta$-positive decay to occur, an electron will be absorbed into the nucleus. The result is the emission of a characteristic $\mathrm{x}$-ray. $\gamma$-decay is the emission of a photon, which has no mass or charge. $\gamma$-emission is usually the result of another decay process. Radioactive decay can be calculated using the half-life of the radionuclide (4).

There are 2 units of radioactivity: becquerels and curies. The becquerel was named after Henri Becquerel, who discovered spontaneous radioactivity. A becquerel is equivalent to 1 disintegration per second. The curie was named after Marie Curie, who discovered radium. One curie is equivalent to $3.7 \times 10^{10} \mathrm{~Bq}$. This is the amount of radioactivity in $1 \mathrm{~g}$ of radium. An easy conversion between becquerels and curies is $1 \mathrm{MBq}=37 \mathrm{mCi}$ (4).

When radiation interacts with matter, excitation or ionization can occur. Excitation causes an electron in the matter to be elevated to a higher-energy shell. Ionization causes the electron in the atom to be removed completely, leaving an ion pair (the electron and the new positively charged atom). Radiation used in nuclear medicine, CT, fluoroscopy, and therapy is ionizing radiation (6).

$\alpha$-particles have a high-linear-energy transfer and will cause a significant number of ionizations, making them useful in therapeutic applications. $\beta$-positive particles (positrons) will interact with a negatively charged electron, causing an annihilation reaction resulting in two $511-\mathrm{keV}$ photons emitting in opposite directions. $\beta$-negative particles will interact with matter by producing bremsstrahlung radiation. Bremsstrahlung radiation is also known as braking radiation. As the $\beta$-negative particle passes close to the nucleus, the attracted forces cause the $\beta$-negative particle to brake, releasing excess energy as bremsstrahlung radiation (an x-ray) (6).

Radionuclides can be produced in 3 basic ways: by a reactor, an accelerator, or a generator. A nuclear reactor uses neutrons to induce fission in uranium. Reactor-produced radionuclides include ${ }^{99} \mathrm{Mo},{ }^{133} \mathrm{Xe},{ }^{131} \mathrm{I}$, and ${ }^{137} \mathrm{Cs}$. Accelerators use charged particles by rapidly accelerating them and slamming them into a target material. A cyclotron is an accelerator that uses 2 magnets (called Dees, for their shape). Cyclotronproduced radionuclides include ${ }^{123} \mathrm{I},{ }^{201} \mathrm{Tl},{ }^{57} \mathrm{Co}$, and many PET radionuclides. A generator has a long-lived parent that decays into a useful radioactive daughter. Generator-produced radionuclides include ${ }^{99 \mathrm{~m}} \mathrm{Tc},{ }^{82} \mathrm{Rb}$, and ${ }^{68} \mathrm{Ga}(4)$.

$\gamma$-rays and $x$-rays differ in their origin. $\gamma$-rays originate in the nucleus, and $\mathrm{x}$-rays originate in the electron cloud. Because there is more energy in the nucleus than in the electron cloud, $\gamma$-rays have the potential to have higher energy than $\mathrm{x}$-rays. However, some $x$-rays do have higher energy than some $\gamma$-rays.

There are 2 types of $\mathrm{x}$-rays produced in an $\mathrm{x}$-ray tube: bremsstrahlung $x$-rays and characteristic x-rays (4). The energy of the $\mathrm{x}$-rays is measured in peak kilovoltage. $\mathrm{x}$-rays are polyenergetic and are produced over a spectrum of energies, whereas $\gamma$-rays are monoenergetic. $\gamma$-rays are measured in kiloelectron volts. The number of $\mathrm{x}$-rays used is measured in milliampere-seconds. The peak kilovoltage is increased for larger patients because the higher-energy $\mathrm{x}$-rays can penetrate the body. The milliampere-seconds is increased to allow for more information (higher quality) on CT but also increases the dose to the patient (4).

A fluoroscopy unit uses a continuous x-ray beam to produce a live image on a monitor. There are several examinations that are performed under fluoroscopy, including barium studies, cardiac catheterization, arthrography, and placement of intravenous catheters. It is also used for lumbar punctures, biopsies, and guided injections into joints. High-dose fluoroscopic procedures are seen in interventional radiology, the cardiac catheterization lab, and the operating room (7).

MRI uses the charge of the atomic nuclei. In fact, MRI was once referred to as nuclear MR. The unit of measurement for a magnetic field is the tesla. One tesla is equal to 10,000 gauss. MRI units are calibrated in the range of 0.5-4 T (8). MRI machines do not produce ionizing radiation.

\section{INSTRUMENTATION REVIEW}

There are 2 main types of instruments that can be used to detect radiation: gas-filled detectors and scintillators. A dose calibrator is a gas-filled instrument known as an ionization chamber. It is located at the lower end of the gas ionization curve (lower voltage). It is useful in measuring high amounts of radiation and is less accurate with low amounts of radiation. A portable ionization chamber, sometimes called a cutie-pie because of its small size, can be used to detect the amount of radiation emitting from a radionuclide therapy patient. A Geiger-Müller (GM) meter is a gas-filled detector located at the upper end of the gas ionization curve (higher 
voltage). It is useful in detecting small amounts of radioactive contamination (9). GM meters are usually calibrated using a high-energy ${ }^{137} \mathrm{Cs}$ source. As such, when they are used to detect lower-energy isotopes (i.e., ${ }^{99 \mathrm{~m}} \mathrm{Tc}$ ), they will overcompensate and the reading will be higher than what is actually there. This makes the instrument sensitive when looking for contamination in the department (4).

Scintillators can be liquid or solid. A liquid scintillator is very efficient and is used for counting low-energy $\beta$-particles. A solid scintillator is much less efficient and found in well counters, $\gamma$-cameras, and PET cameras. Well counters are useful in detecting removable contamination. The efficiency of well counters varies. Therefore, it is important to correct the counts by the instrument's efficiency and report all values in disintegrations, not counts (9).

Radiation detection instruments need to be calibrated and checked regularly. A GM meter should be calibrated annually using a high-activity ${ }^{137} \mathrm{Cs}$ source. It should also be checked for a response each day before use. The daily check should include a battery check, annual calibration expiration date check, and source check. Some meters have a dedicated source. The reading should be within $\pm 20 \%$ of the expected reading (4).

Four tests are required for a dose calibrator (Table 2). An accuracy test is performed annually and includes measuring a source with a known amount of radioactivity. A minimum of 2 sources should be used. The most common sources are ${ }^{137} \mathrm{Cs},{ }^{133} \mathrm{Ba}$, and ${ }^{57} \mathrm{Co}$. The measured amount is compared with the known amount. Accuracy should be within $\pm 10 \%$. A constancy test, a test of precision, is performed daily. The expected value comes from either previous readings or calculated readings. Precision should be within $\pm 10 \%$ of the expected reading. A geometry test is performed to ensure that radioactivity in different geometric variations (i.e., different volumes or differently sized syringes or vials) is precise. This test is done when a new dose calibrator is brought into the department, when repairs have been made, or when a new size of syringe or vial is introduced. Measurements should be within $\pm 10 \%$ of the expected value. A linearity test is performed quarterly to ensure that the dose calibrator is accurate over a wide range of activities. This test can be done with the decay method or the attenuation sleeve method. Readings should be within $\pm 10 \%$ of the expected value (4).

\section{MEASURING RADIATION}

There are several units used to measure radiation exposure and dose (Table 3). The unit of exposure is the roentgen. This is the unit used on GM meters. One roentgen is equivalent to
$2.58 \times 10^{-4} \mathrm{C}$ per kilogram. The unit of exposure measures ionizations in air (i.e., gas-filled detectors, such as the GM meter) and is useful for photons only. The unit of absorbed dose is the rad or gray ( $100 \mathrm{rad}=1 \mathrm{~Gy}$ ). This unit measures the amount of energy imparted on a material. It is useful for photons and particulate radiation but does not distinguish between the two. The sievert or rem $(100 \mathrm{rem}=1 \mathrm{~Sv})$ is a unit of equivalent dose. This unit also measures the amount of energy imparted on a material but differentiates between different types of materials by multiplying by the quality factor for that type of radiation. When rads or grays are multiplied by the quality factor, the unit changes to rems or sieverts (unit of equivalent dose). The quality factor for x-rays, $\gamma$-rays, and $\beta$-particles all have a quality factor of 1 . The unit of effective dose is also rems or sieverts but takes into consideration what part of the body was exposed. If the whole body was exposed (as assumed with whole-body badges), the tissue weighting factor is 1 . If only part of the body was exposed, the tissueweighting factor is less than 1 (4).

Occupational dose should be measured for any worker likely to receive $10 \%$ of the annual limit (5 Sv or 500 mrem) (1). This dose can be monitored using radiation badges (whole-body and rings). Commonly, a whole-body dosimeter is worn on the body between the neck and waist. The badge is a made of an optically stimulated luminescence detection material. Ring badges containing a thermoluminescence dosimeter chip are worn on the dominant hand, facing the palm to measure extremity exposure. Film dosimeters and pocket dosimeters can also be used to measure exposure to occupational workers (4).

Bioassays are used to detect an individual's exposure to internal radiation. The most common bioassay is a thyroid count done to detect an individual's exposure to ${ }^{131} \mathrm{I}$. Other bioassays include a urine sample, nasal swab, and wholebody counting. The occupational worker's total dose is the sum of the deep dose equivalent (external dose) and committed dose equivalent (internal dose) (1).

Individuals can be internally exposed by ingestion, absorption, or inhalation. The annual limit of intake results in an effective dose of 5 rem (0.05 sievert). Each radiopharmaceutical has a level that leads to 1 annual limit of intake. If a dose is inhaled, the air concentration can be calculated for different radionuclides to produce a derived air concentration. Although ${ }^{133} \mathrm{Xe}$ is airborne, the preferred method of determining workers' exposure to ${ }^{133} \mathrm{Xe}$ is to use the deep dose equivalent, which is a measure of external dose (1).

TABLE 2

Dose Calibrator Quality Control

\begin{tabular}{|c|c|c|c|c|}
\hline Test & Purpose & Source & Frequency & Passing level \\
\hline Accuracy & Accuracy & ${ }^{137} \mathrm{Cs},{ }^{57} \mathrm{Co},{ }^{133} \mathrm{Ba}$ & Annually & $\pm 10 \%$ \\
\hline Constancy & Precision & ${ }^{137} \mathrm{Cs}$ or ${ }^{57} \mathrm{Co}$ & Daily, before use & $\pm 10 \%$ \\
\hline Linearity & Precise over range of activities & ${ }^{99 m}$ Tc or ${ }^{18} \mathrm{~F}$ & Quarterly & $\pm 10 \%$ \\
\hline Geometry & Precise over different configurations/volumes & $99 \mathrm{mTc}$ & New or after repairs & $\pm 10 \%$ \\
\hline
\end{tabular}


TABLE 3

Radiation Measurement Units

\begin{tabular}{lccc}
\hline \multicolumn{1}{c}{ Measurement } & U.S. unit & SI unit & Conversion \\
\hline Activity & Curie & Becquerel & $1 \mathrm{mCi}=37 \mathrm{MBq}$ \\
lonizations in air & Roentgen & C $/ \mathrm{kg}$ & $1 \mathrm{R}=2.59 \times 10^{-4} \mathrm{C} / \mathrm{kg}$ \\
Absorbed dose & Rad & Gray & $100 \mathrm{rad}=1 \mathrm{~Gy}$ \\
Equivalent dose (absorbed dose times radiation quality factor) & Rem & Sievert & $100 \mathrm{rem}=1 \mathrm{~Sv}$ \\
Effective dose (equivalent dose times tissue weighting factor) & Rem & Sievert & $100 \mathrm{rem}=1 \mathrm{~Sv}$ \\
\hline
\end{tabular}

In addition to monitoring of an individual's exposure, room monitoring is useful to reduce exposure. Ambient dose rate surveys are performed using a GM meter to detect contamination or sources of radioactivity in an area. Trigger levels for ambient dose rate should be set at $50 \mu \mathrm{Sv} / \mathrm{h}$ $(5 \mathrm{mrem} / \mathrm{h})$ for restricted areas and $2 \mu \mathrm{Sv} / \mathrm{h}(0.2 \mathrm{mrem} / \mathrm{h})$ for unrestricted areas (1). Wipe tests are performed using a well counter to identify areas where there is removable contamination. A trigger level of 22,000 dpm/100 $\mathrm{cm}^{2}$ should be established (1).

Sealed sources should also be monitored. A biannual inventory should be maintained, and removable contamination (leak test) should be measured. All sealed sources should be inventoried and leak-tested, with the following exceptions: sources with a half-life of less than $30 \mathrm{~d}$, sources containing gaseous material, sources containing $3,700 \mathrm{kBq}(100 \mu \mathrm{Ci})$ or less, and sources in storage and not being used (1). The leak test result should be less than $185 \mathrm{~Bq}(0.005 \mu \mathrm{Ci})(1)$. To use a well counter to perform a leak test, a minimum detectable activity needs to be established on that well counter. The minimum detectable activity needs to be less than $185 \mathrm{~Bq}(0.005 \mu \mathrm{Ci})$.

\section{RADIATION PROTECTION AND ALARA}

A deterministic effect to radiation occurs when a threshold is reached. The severity of the effect increases with dose. Examples of deterministic effects are skin erythema and cataracts. Stochastic effects are random effects, and their prevalence is more likely with a higher dose (4).

The 3 basic methods of radiation safety are time, distance, and shielding. Limiting time near a radiation source will lower exposure. Using distance (using the inverse square law) can reduce exposure. The inverse square law states that if you double your distance from the source, you reduce the exposure rate to a quarter of the original value. Shielding can be used to reduce exposure. This reduction can be calculated using the linear attenuation coefficient. Each shielding material has a half-value layer for each radionuclide. The linear attenuation coefficient is 0.693 /half-value layer (4).

Radiation workers must not exceed annual limits. The annual limits for a radiation worker are $0.05 \mathrm{~Sv}$ (5 rem) to the whole body, $0.15 \mathrm{~Sv}$ (15 rem) to the lens of the eye, and $0.5 \mathrm{~Sv}$ (50 rem) to an extremity or skin of the whole body. Minors (those under the age of $18 \mathrm{y}$ ) have an annual limit of $10 \%$ of the adult limit. Radiation workers who have voluntarily declared pregnancy have a $0.05 \mathrm{~Sv} / \mathrm{mo}(50 \mathrm{mrem} / \mathrm{mo})$ or 0.5 $\mathrm{Sv}$ (500 mrem) over the gestational period for the fetus (4).
The public must also be protected from radiation exposure. The limits to the public are less than $0.02 \mathrm{mSv}$ (2 mrem) in any hour. This is a total dose in a 1-h period, not a dose rate. In addition, it must be less than $1 \mathrm{mSv}(100 \mathrm{mrem})$ in $1 \mathrm{y}$. This exposure does not include doses received from radioactive patients (6).

Radiation workers must be notified at least annually of their radiation exposure. Records must be kept by the employer for the entire length of the license. Employers should follow the ALARA (as low as reasonably achievable) principle to keep exposure low. Notifications should be provided to workers who exceed $10 \%$ of the quarterly limit (0.125 Sv or $125 \mathrm{mrem})$. In addition, for workers who exceed $30 \%$ of the quarterly limit $(0.375 \mathrm{~Sv}$ or $375 \mathrm{mrem})$, a plan of action should be implemented to reduce exposure (1).

Restricted areas are those where people may be exposed to radiation or radioactive materials. A restricted area should have controlled access. Doors to restricted areas should be locked when a direct line of sight is unavailable. In addition, signs indicating the level or type of radiation should be posted. The following signs are used: "Caution, Radiation Area," "Caution, High Radiation Area," "Grave Danger, Very High Radiation Area," "Caution, Airborne Radioactivity Area," and "Caution, Radioactive Materials" (4).

Currently, there are no regulations on the amount of radiation exposure to patients. Radiation workers should strive to use the appropriate dose for the examination. The "Image Gently" and "Image Wisely" campaigns are useful resources for identifying appropriate doses. Many factors can affect patient dose, including the half-life and biodistribution of the radiopharmaceutical. Patients should be evaluated to determine pregnancy or lactation status. Patients who are lactating (even if they discontinue breast feeding) may have increased breast uptake. The risks and benefits must be explained to the patient before the procedure is performed.

CT dose is measured in CT dose index (CTDI). This is the dose measured in a phantom and can be used to evaluate risk to populations, not to individual patients. The CTDI is a summed dose based on peak kilovoltage, milliampereseconds, collimation, and rotation speed (4). Dose-length product is the CTDI multiplied by the length of the scan. Both CTDI and dose-length product are measured in mGy. There are 2 CTDI phantoms. The large (body) phantom is $32 \mathrm{~cm}$ in diameter. The small (head) phantom is $16 \mathrm{~cm}$ in diameter. Both CTDI and dose-length product are a measure 
of how much radiation is being used, not how much is absorbed by the individual patient (10).

Peak kilovoltage is usually set between 80 and $140 \mathrm{kVp}$. Milliampere-seconds is usually set between 50 and 400 mAs. Automatic exposure control is used to adjust the tube current as the body is being scanned. Different areas of the body have different densities. Automatic exposure control adjusts accordingly (10).

The effective dose (in $\mathrm{mSv}$ ) can be calculated for adults by multiplying the dose-length product by the $\mathrm{k}$ factor. The $\mathrm{k}$ factor is found in the American Association of Physicists in Medicine report 96 (11). In addition, patient size will make a difference when identifying the dose to the patient. Sizespecific dose estimates are factors that account for differences in patient size. Smaller patients will have an underestimated CTDI, and larger patients will have an overestimated CTDI. Size-specific dose estimates can be found in American Association of Physicists in Medicine Report 204 (12).

There are 4 main metrics for fluoroscopy dose: fluoroscopy time, air kerma, dose-area product, and peak skin dose. Fluoroscopy time is the amount of time a patient is exposed. Air kerma is expressed in $\mathrm{J} / \mathrm{kg}$ or grays. It is a quantity of absorbed dose. Dose-area product is the absorbed dose multiplied by the area radiated and is expressed in gray- $\mathrm{cm}^{2}$. Peak skin dose is the highest dose to any portion of the patient's skin during a procedure (13).

The biggest safety risks associated with MRI are that objects can become projectiles and slam into the magnet, burns can be created by energy deposition, and hearing loss can occur because of the loud noises produced by the scanner (14). To prevent unauthorized access into the MRI suite, the ACR recommends 4 safety zones. Zone 1 includes an area that is open to the general public. This area is less than 5 gauss $(0.5 \mathrm{mT})$. Zone 2 is also a public area, but it is an intermediate area that separates zone 1 from zones 3 and 4 and is typically where the technologist will perform patient screening. Zone 3 is the area near the magnet room. Only screened individuals may enter zone 3. Zone 4 is the magnet room itself (15).

\section{EMERGENCY PROCEDURES AND ADVERSE EVENTS}

Several emergency procedures are in place for individuals working with radioactive materials. Major and minor radiation spills are handled differently. Many times, the RSO will not be involved with a minor spill because it can be handled in the department (10). Other examples of emergency situations include radiotherapy patients requiring emergency care, as well as the unexpected death of a radiotherapy patient. RSOs may also be called on to lead emergency responses for radiologic events.

The term misadministration has been replaced with medical event. Technologists thought a misadministration occurred when the wrong radiopharmaceutical was administered even if the dose was less than the 5-rem threshold. However, by definition this would not be a misadministration. It would be an error. It still requires reporting, but not to the NRC. A medical event occurs when an error and an excess exposure occur at the same time. An error occurs when the patient, amount, or route is wrong. An excess exposure occurs when a dose is given that results in delivery of more than $0.05 \mathrm{~Sv}$ (5 rem) to the whole body, $0.5 \mathrm{~Sv}$ (50 rem) to an organ or tissue, or $0.5 \mathrm{~Sv}$ (50 rem) to the skin. Therapies are examples of excess exposures. When an excess exposure and an error occur with the same administration, a medical event has occurred. The most common medical event in a nuclear medicine department occurs with the administration of ${ }^{131} \mathrm{I}$. A medical event must be reported to the NRC within 1 calendar day after discovery of the event (1).

\section{CONCLUSION}

Technologists seeking to assume the responsibilities of RSO or associate RSO should seek multiple references when preparing for that role. In addition to reading and understanding the regulations, at least $1 \mathrm{y}$ of experience should be sought under the supervision of an RSO.

\section{DISCLOSURE}

No potential conflict of interest relevant to this article was reported.

\section{REFERENCES}

1. Siegel JA. Guide for Diagnostic Nuclear Medicine and Radiopharmaceutical Therapy. Reston, VA: Society of Nuclear Medicine and Molecular Imaging; 2004.

2. 49 CFR 172.704 Training Requirements. Washington, DC: Department of Transportation; 2015.

3. Manual Transportation of Radioactive Material. Chattanooga, TN: USNRC Technical Training Center; 2003.

4. Waterstram-Rich KM, Gilmore D. Nuclear Medicine and PET/CT: Technology and Techniques. St. Louis, MO: Elsevier Health Sciences; 2016.

5. 49 CFR 173.421 Excepted Packages for Limited Quantities of Class 7 (Radioactive) Materials. Washington, DC: Department of Transportation; 2014.

6. Cherry SR. Physics in Nuclear Medicine. 4th ed. Philadelphia, PA: Elsevier; 2012.

7. NMTCB radiation safety examination content outline. NMTCB website. http:// nmtcb.org/documents/NMTCB-Radiation-Safety-Examination-Content-Outline2017-FINAL.pdf. Published 2017. Accessed October 1, 2018.

8. Faulkner W. Rad Tech's Guide to MRI: Basic Physics, Instrumentation, and Quality Control. Hoboken, NJ: Wiley-Blackwell; 2001.

9. Prekeges J. Nuclear Medicine Instrumentation. Sudbury, MA: Jones and Bartlett Publishers; 2012.

10. Lee K. Basic Science of Nuclear Medicine: The Bare Bone Essentials. Reston, VA: Society of Nuclear Medicine and Molecular Imaging; 2015.

11. McCollough C. AAPM Report No. 95 The Measurement, Reporting and Management of Radiation Dose in CT 2008.

12. AAPM Report No. 204: Size-Specific Dose Estimates (SSDE) in Pediatric and Adult Body CT Examinations. College Park, MD: American Association of Physicists in Medicine; 2011.

13. Miller DL, Balter S, Dixon RG, et al. Quality improvement guidelines for recording patient radiation dose in the medical record for fluoroscopically guided procedures. J Vasc Interv Radiol. 2012;23:11-18.

14. Panych LP, Madore B. The physics of MRI safety. J Magn Reson Imaging. 2018;47:28-43.

15. Kanal E, Barkovich AJ, Bell C, et al.; Expert Panel on MR Safety. ACR guidance document on MR safety practices 2013. J Magn Reson Imaging. 2013;37:501-530. 